Recibido: 30/03/2016

Aprobado versión definitiva: 28/04/2016

\title{
ANÁLISIS SOCIO-HISTÓRICO COMPARADO SOBRE LAS FORMAS DE PARTICIPACIÓN POLÍTICA EN VENEZUELA Y ECUADOR
}

\author{
JOSÉ ISMAEL CRIADO AGUILERA ${ }^{1}$
}

\section{RESUMEN}

Se analiza de forma comparativa, cualitativa e interdisciplinar la situación socio-política y económica en Venezuela y Ecuador. Se define el concepto teórico de participación política, para estudiar las formas de participación democrática y electoral en el siglo XX, y se contrastan con las nuevas formas de democracia participativa y directa plasmadas en sus Constituciones. Se estudian los datos empíricos recogidos en índices internacionales, y el desarrollo real de estas nuevas formas para valorarlas y esbozar sus líneas futuras de investigación. Hallamos que clientelismo y partidismo polarizan la participación política en Venezuela, mientras que en Ecuador son cuestiones fundamentales la justicia constitucional y la plurinacionalidad indígena.

Palabras clave: Democracia directa, Democracia participativa, Procesos constituyentes, Derechos políticos.

\footnotetext{
${ }^{1}$ Graduado en Ciencias Políticas y de la Administración de la Universidad de Granada (España). Afiliado al Centro de Estudios Políticos y Constitucionales - Ministerio de la Presidencia- Gobierno de España (España). Avenida Bulevar, 170, 2 A - El Ejido. Código postal 04700, Almería, España. Correo electrónico: elejidojc@gmail.com
} 


\section{SOCIO-HISTORICAL COMPARATIVE ANALYSIS ABOUT POLITICAL PARTICIPATION WAYS IN VENEZUELA AND ECUADOR.}

\section{ABSTRACT}

There is analysed in an interdisciplinary, qualitative and comparative way the economic and socio-politic situation in Venezuela and Ecuador. There is defined politic participation theoretical concept, in order to study XX century's electoral and democratic participation ways, and it contrasts with new participative and direct democracy ways. It studies empirical dates recollected on international indexes, and the real development of these new ways to value them and drafting their future investigation lines. We find patronage logics and partisanship polarize political participation in Venezuela, meanwhile in Ecuador constitutional justice and indigenous plurinationality are key questions.

Key words: Participative democracy, Direct democracy, Constituent processes, Political rights.

\section{Introducción: Objetivos y líneas metodológicas}

El objetivo general del presente artículo de investigación es analizar y explicar la evolución de la participación política, en grado y formas, en dos países de América Latina como son Ecuador y Venezuela. Para ello, emplearemos una metodología cualitativa, a través del análisis y revisión documental como técnica de investigación fundamental, desde un enfoque comparativo. Asimismo, el carácter interdisciplinar del proceso se compondrá de elementos de análisis de tipo histórico, jurídico, sociológico y politológico. Esto aportará y enriquecerá la investigación para tomar en adecuada consideración los elementos transcendentales que vertebran los andamiajes políticos en ambos países. Con este marco metodológico, y apoyando el desarrollo de la investigación en referencias empíricas centrales, pretendemos cumplir con tres objetivos específicos que guiarán consecutivamente las etapas de nuestro análisis:

1) Cartografiar con precisión, de forma crítica y rigurosa, la situación política actual en Ecuador y Venezuela. 
2) Analizar las formas contemporáneas de participación política de las sociedades ecuatoriana y venezolana hasta llegar al siglo XXI, en que tuvieron lugar los últimos procesos constituyentes. Esto con el fin de construir una base histórica que nos permita comprender en su contexto los cambios sociales acaecidos en ambas naciones durante las últimas décadas.

3) Definir el concepto de participación política e indagar en profundidad sobre las nuevas formas de la misma que se vienen desarrollando normativa y empíricamente en Ecuador y Venezuela. De esta forma se podrán establecer unas conclusiones sólidas que nos permitan avanzar en la investigación futura sobre estas cuestiones.

Para abordar tales objetivos, llevaremos a cabo una descripción bidireccional esquemática y entrecruzamiento interpretativo.

\section{Breves esbozos sobre las características socio-económicas e historia política de ambos países. Situación política actual; tras los procesos constituyentes de 1999 y 2008}

Existe un amplio consenso respecto a que América Latina ha cambiado profundamente en las últimas décadas tanto en lo social, como en lo económico y en lo político. Es razonable afirmar que nos hallamos en una nueva época histórica marcada, entre otras cosas, por la superación del pasado autoritario a través de la consolidación de sistemas democráticos efectivos en la gran mayoría de los países del continente. En los últimos treinta años, como indican Bonometti y Ruiz Seisdedos (2010), "América Latina ha experimentado la transición y la consolidación democrática. Podemos considerar eso como un gran avance para la región, en el sentido de que la democracia es la forma más idónea para asegurar el desarrollo humano" (p.29).

Sin embargo, en relación al plano socio-económico, la pobreza crónica que sufre una parte de la población en estos países $^{2}$, nos genera incertidumbre acerca

\footnotetext{
2 A pesar de que la pobreza se redujo casi a la mitad en Ecuador de 1999 a 2013 (https://www.cia.gov/library/publications/the-world-factbook), quedando en el 25,6\%, y en
} 
de la estabilidad socio-política democrática en las naciones latinoamericanas, y pueden condicionar la participación política en sus grados y formas. Sumado a esta situación, se encuentra el actual estancamiento de la economía a nivel regional, que decreció un 0,9\% en 2015, según los indicadores del Banco Mundial. A pesar de no ser objeto específico de estudio en este trabajo, somos conscientes que estos tres planos (económico, social y político) están estrechamente interrelacionados y son, en buena medida, interdependientes.

En cuanto al plano político, para abordar los objetivos de esta investigación consideramos conveniente realizar una previa comparativa general de la situación política actual en Venezuela y Ecuador. Para tales fines, inexorablemente hemos de comenzar a trabajar a partir de los recientes procesos constituyentes que han tenido lugar en ambas naciones. Las Cartas Magnas con las que se dotaron Venezuela (1999) y Ecuador (2008) vertebran los procesos, métodos y mecánicas de participación política actuales, tanto en el plano normativo como también en buena medida programático.

Ambos procesos estuvieron marcados por métodos socio-políticos inclusivos y participativos (esto es, protagonizados por actores que en su mayoría no estaban integrados en el sistema o jugaban un papel marginal hasta entonces) de corte comunitarista. Sus objetivos fueron profundamente transformadores de las estructuras de poder establecidas. Por tales razones, se auto-denominaron revolucionarios: Revolución Bolivariana en Venezuela (Gott, 2005) y Ciudadana en Ecuador (Núñez Sánchez, 2014).

La participación política la entendemos como un elemento esencial en la vida democrática. Como indica Uriarte (2008), se puede definir de forma general como toda actividad de la ciudadanía dirigida a intervenir, de forma directa, indirecta, convencional o no convencional, en la política estatal. De la multiplicidad de definiciones que existen de este concepto, cabe extraer la presencia de tres

Venezuela bajó del $42,8 \%$ al $27,3 \%$ en el mismo período (http://www.ine.gov.ve). No obstante, estas tasas siguen siendo muy elevadas. 
elementos comunes que son la acción, la influencia, y lo político como marco epistemológico.

La participación ciudadana en política jugó un papel central en ambos procesos constituyentes y en las configuraciones institucionales subsiguientes. Otro hito fundamental que enmarca también esta tendencia participativa es la descentralización del poder político, cuestión a la que anteriormente no se consideraba con la importancia que adquirió en sendos procesos constituyentes ${ }^{3}$. En ambos Estados existían niveles de descentralización administrativa, pero la relativa al ámbito político estaba muy poco desarrollada, sujeta al control centralista del Estado.

En el caso de Venezuela existían las gobernaciones estatales, cuyos jefes eran designados desde Miraflores, al igual que sucedía con los municipios. Ambos procedimientos de designación estuvieron vigentes hasta 1984, en el caso de los municipios, y 1989, año en que se realizaron las primeras elecciones a los cargos políticos de los Estados federados. En el caso de Ecuador, las entidades seccionales y locales comenzaron a elegirse por el voto popular en 1978- No obstante, como en el caso venezolano, la arraigada mentalidad centralista y la dependencia (principalmente de recursos económicos) del poder central, limitaron el impacto democrático de esta descentralización, ralentizando además su desarrollo.

Paralelamente, ambos procesos constituyentes estuvieron marcados por un ideario basado en la recuperación de la soberanía popular y la independencia nacional, cuya interpretación práctica se tradujo en una mayor intervención del Estado, de las instituciones públicas en la vida económica. Comprobamos que, salvando las diferencias profundas que existen entre Venezuela y Ecuador, los procesos políticos emprendidos en ambos países poseen un trasfondo ideológico compartido que se basa en la justicia social. Este principio se ha venido materializando en las diferentes medidas sociales que han implementado sus

\footnotetext{
${ }^{3}$ Sin embargo, paradójicamente la CRBV de 1999 eliminó el Senado, institución fundamental de representación territorial en la teoría política del federalismo.
} 
respectivos gobiernos (tanto laborales y asistencialistas, como de aumento del gasto público en materias como la educación o la sanidad, entre otras ${ }^{4}$ ).

Para completar el primer objetivo específico de la investigación, es menester desplazarnos del plano socio-económico y teórico-ideológico que veníamos tratando, y centrarnos en la situación política actual, cotidiana de ambas naciones. En ella hallamos en general un deterioro de la dinámica desarrollista y estable de los primeros años revolucionarios, especialmente en Venezuela. En el caso de Ecuador, la situación política se encuentra mucho más estable y consolidada la legitimidad del Gobierno, lo cual se refleja en la sólida victoria electoral del Presidente Correa con un $56,6 \%$ del voto (quedando el segundo candidato a más de veinte puntos porcentuales de diferencia) en las pasadas elecciones de 2013. Ello, a pesar de que también existen profundas dificultades y conflictividad política ${ }^{5}$, deterioro de la imagen del Presidente y el Gobierno en cuanto a su política mediática, y creciente oposición de sectores indígenas y ecologistas ante políticas como el proyecto petrolífero en el Yasuní (territorio amazónico protegido).

Sin embargo, en Venezuela, la tesitura socio-política ha empeorado notablemente tras la muerte del Presidente Hugo Chávez a principios de marzo del 2013. Apenas cinco meses después de ser reelecto con el $55,25 \%$ del voto y a una distancia considerable, de más de diez puntos sobre su rival, Henrique Capriles, se convocaron nuevos comicios presidenciales como establece la Constitución en su artículo 233. La elección realizada el 14 de abril de ese año otorgó la victoria al candidato oficialista (designado por el mismo Chávez en su último mensaje televisado a la nación) Nicolás Maduro, con el 50,61\% frente al $49,12 \%$ de Capriles. Se trata de un triunfo con un margen mínimo, de poco más de

\footnotetext{
4 Para profundizar en estas cuestiones, ofrecemos los siguientes enlaces que relatan las "conquistas revolucionarias" en ambos países, recomendando una lectura crítica dada la abierta posición pro-oficialista de la fuente, Telesur. http://www.telesurtv.net/telesuragenda/Venezuela-16anos-de-Revolucion-20150202-0019.html; http://www.telesurtv.net/news/Cuanto-ha-cambiadoEcuador-con-la-Revolucion-Ciudadana--20150115-0097.html.

${ }^{5}$ El caso más conocido sucedió el 30 de septiembre de 2010 , que las autoridades calificaron como secuestro del Presidente de la República, intento de magnicidio y de golpe de Estado.
} 
doscientos mil votos, que hizo ver de forma clara la seria competencia de una alternativa de Gobierno por primera vez en 15 años. Además, fueron unos resultados cuestionados y deslegitimados en todo momento por la oposición.

Las razones fundamentales de la pérdida, en poco más de seis meses, de más de medio millón de votos entre la elección de Chávez y la de Maduro fueron, obviamente la carencia de un liderazgo consolidado y un carisma como el de Chávez. También, por los graves problemas socio-económicos que se han venido acentuando de forma extraordinaria en los últimos tres años. Los más relevantes son, en primer lugar, la corrupción política y económica; la percepción de la misma entre la población es muy alta ${ }^{6}$, concibiendo la ciudadanía que existen importantes niveles de corrupción en casi todos los estratos de la sociedad, lo que debilita la confianza en la capacidad de gestión del Estado, así como la legitimidad global de las instituciones.

En segundo lugar, la inseguridad ciudadana; Venezuela es el segundo país del mundo con mayor tasa de homicidios ${ }^{7}$, aparte de otras formas de criminalidad como los robos, las agresiones, y demás formas de delincuencia que se amparan en una sensación de impunidad criminal. Este panorama, retroalimenta a nuestro juicio un estado de psicosis colectiva dramático, difícilmente solucionable a priori. Todo ello se suma a la grave crisis de escasez y desabastecimiento de alimentos y todo tipo de insumos, lo que incide de forma trágica en la gran mayoría de los sectores socio-económicos y los servicios públicos, entre los que destaca por su carácter esencial la sanidad, dada la falta de medicamentos, equipamientos hospitalarios, etc.. Estos fenómenos se retroalimentan con la galopante tasa de inflación, que llegó este pasado año a superar el $180 \%{ }^{8}$ y situarse como la más alta del mundo.

\footnotetext{
6 Situándose Ecuador y Venezuela, según el índice que elabora Transparencia Internacional (http://www.transparency.org/cpi2015\#results-table), en los puestos 107 y 158, respectivamente, de 167 países estudiados, siendo los últimos los países donde existe mayor percepción de corrupción. ${ }^{7}$ Fuente de referencia: http://observatoriodeviolencia.org.ve/

8 Para ampliar estas cuestiones, recomendamos el siguiente compendio noticiario del diario ecuatoriano El Universo: http://www.eluniverso.com/noticias/2016/02/21/nota/5416287/escasezparte-cotidiano-venezuela
} 
Estos dos conjuntos de factores inciden muy negativamente en el conjunto del país, así como en la participación política. A ello se pueden añadir otros problemas también muy importantes como la fuerte polarización política, que dificulta con creces la gobernabilidad de la nación. Además de la perspectiva extendida de que existe una deriva autoritaria y burocratista del Gobierno.

Finalmente, la arraigada mentalidad rentista, combinada con el descenso brutal de los precios internacionales del petróleo (esto también azota duramente a Ecuador, ya que, como en Venezuela, se trata de la principal fuente de ingresos del Estado), incide en una situación de inestabilidad económica muy grave. El potente auge del mercado negro de divisas en Venezuela -que tiene mucho peso debido al estricto control de cambio fijado por el Estado en la última década-) alimenta la inflación, que galopa descontrolada en su distorsión de precios y pérdida enorme de poder adquisitivo de la ciudadanía. La vida cotidiana de la población también se ve afectada por la regulación de precios y la subvención de productos básicos, que generan una conjunción de especulación, contrabando y escasez crónica de productos de primera necesidad, así como colas, racionamiento de alimentos específicos como las harinas, aceites, lácteos, medicamentos.

En Ecuador sin embargo, el empleo del dólar como moneda oficial del país, viene asegurando una mayor estabilidad económica debido a que se trata de una divisa fuerte en los mercados mundiales, y está sujeta a menores distorsiones económicas ${ }^{9}$. Todos los factores socio-políticos y económicos mencionados se reflejan en las diferenciadas tendencias de consolidación y profundización de la democracia que atraviesan estos dos países. Tal como lo indican instituciones como el Índice de Desarrollo Democrático de América Latina (IDD-LAT), avanzan positivamente en Ecuador y retroceden en Venezuela.

\footnotetext{
9 Si bien es cierto que la carencia de soberanía monetaria también conlleva una serie de limitaciones de primer orden.
} 


\section{Las tradicionales formas de participación política: Centralismo y representatividad}

Es amplio el consenso socio-académico respecto a que los modelos de democracia liberal-representativa existentes en Venezuela y Ecuador durante la segunda mitad del siglo $\mathrm{XX}$ se fueron deteriorando progresivamente hasta agotarse, debido a la pérdida enorme de legitimidad y apoyo popular. La incapacidad para satisfacer las demandas democráticas, especialmente de contenido económico-social (pero también de tipo político, de género e identitario), así como la exclusión de amplios sectores de la población del proceso político (aspectos simbióticos), resultaron finalmente letales para la legitimidad constitucional.

Como indica López Maya (2012) refiriéndose al caso de Venezuela:

En este régimen político, el poder popular se expresa, ya no en la calle y/o de modo espontáneo e informal, sino principalmente a través del sufragio universal, directo y secreto, que se vuelve a consagrar en la Constitución de 1961, donde los partidos políticos se asumen como mediadores casi exclusivos entre sociedad y Estado (p.59).

La partitocracia que progresivamente hizo suyas las instituciones del Estado fue la principal causante de esta situación. La corrupción endémica del sistema fue poco a poco minando la credibilidad institucional hasta que llegó a ser una situación irreversible. Algunos casos son: el clientelismo más raso que llegó hasta sus instituciones centrales (cabe citar el procesamiento judicial del que fuera Presidente, Carlos Andrés Pérez, en Venezuela, un caso paradigmático de esta realidad); las profundas fallas en cuanto a la separación e independencia de los poderes del Estado; la violación de los derechos fundamentales mediante las recurrentes suspensiones de garantías constitucionales como respuesta a la conflictividad social; y la represión de movimientos populares, universitarios, indígenas más en el caso de Ecuador.

En el caso ecuatoriano, la gravedad de la situación socio-política también hizo crecer, en los márgenes del sistema una serie de movimientos sociales, 
políticos, igual que lo fue el Movimiento Bolivariano Revolucionario en Venezuela. Estos movimientos iban desde las tendencias progresistas identificadas con la izquierda clásica hasta los movimientos indígenas que reclamaban además el reconocimiento identitario que les había sido negado a lo largo de toda la historia. La crisis de representación llevó en este caso a emprender un proceso constituyente en 1998, que dio lugar a una Carta Magna más avanzada, inclusiva, pero que como veremos no tuvo el efecto que se esperaba. La inestabilidad política y la inefectividad de los derechos sociales que reclamaban los sectores más desfavorecidos, hicieron que la Constitución se deslegitimara poco después de comenzar a aplicarse, y que de nuevo fueran articulándose movimientos en sus márgenes que abogaban por un nuevo proceso constituyente, verdaderamente inclusivo y con una concepción integral de los derechos sociales y políticos.

En el caso de Venezuela, la crisis orgánica de la democracia fundada con la Constitución venezolana de 1958 se torna irreversible a final de la década de los '80 y durante toda la década posterior. Para entonces, ya las formas tradicionales de hacer política estaban ampliamente denostadas para importantes sectores de estas naciones latinoamericanas. Lo anterior se demuestra con el abrupto descenso que sufrió el mecanismo de participación política por excelencia, el sufragio, como podemos comprobar en las series históricas de participación electoral de ambos países que se muestran en los siguientes cuadros: 


\section{Cuadro 1. Datos de participación en elecciones presidenciales}

y parlamentarias en Venezuela (1958-2015).

\begin{tabular}{|c|c|c|}
\hline Año/Elecciones & Presidenciales & Legislativas \\
\hline $\mathbf{1 9 5 8}$ & $92,15 \%$ & $93,42 \%$ \\
\hline $\mathbf{1 9 6 3}$ & $92,21 \%$ & $90,84 \%$ \\
\hline $\mathbf{1 9 6 8}$ & $96,73 \%$ & $94,51 \%$ \\
\hline $\mathbf{1 9 7 3}$ & $96,52 \%$ & $96,52 \%$ \\
\hline $\mathbf{1 9 7 8}$ & $87,55 \%$ & $87,56 \%$ \\
\hline $\mathbf{1 9 8 3}$ & $87,75 \%$ & $87,75 \%$ \\
\hline $\mathbf{1 9 8 8}$ & $81,92 \%$ & $81,65 \%$ \\
\hline $\mathbf{1 9 9 3}$ & $60,16 \%$ & $60 \%$ \\
\hline $\mathbf{1 9 9 8}$ & $63,45 \%$ & $52,65 \%$ \\
\hline $\mathbf{2 0 0 0}$ & $56,31 \%$ & $56,55 \%$ \\
\hline $\mathbf{2 0 0 6 / 2 0 0 5}$ & $74,69 \%$ & $25,26 \%$ \\
\hline $\mathbf{2 0 1 2 / 2 0 1 0}$ & $82,28 \%$ & $60,42 \%$ \\
\hline $\mathbf{2 0 1 3 / 2 0 1 5}$ & $79,64 \%$ & $74,17 \%$ \\
\hline
\end{tabular}

Fuente: International Institute for Democracy and Electoral Assistance (International IDEA).

Tanto para las parlamentarias como para las elecciones presidenciales en Venezuela, hallamos un punto de inflexión fundamental en el año 1993, en el cual los datos de participación bajaron a sus mínimos históricos apenas logrando el $60 \%$ de la participación ciudadana. Estas tendencias se logran invertir, con ciertas excepciones coyunturales como la motivada por la estrategia abstencionista de la oposición venezolana en las elecciones legislativas de 2005, hasta llegar a máximos solo alcanzados cuando el voto era obligatorio (hasta 1993 para las presidenciales y 1998 para las legislativas), en las dos últimas presidenciales (que rondan el $80 \%$ de participación). También, en las parlamentarias, ya que tras el $66,42 \%$ de 2010 (el mejor dato desde 1988), en las recientes de 2015 ha participado más del $74 \%$ del electorado ${ }^{10}$.

\footnotetext{
${ }^{10}$ Datos disponibles en:

http://www.bbc.com/mundo/noticias/2015/12/151204 venezuela_parlamentarias oposicion chavis $\underline{\text { mo dp }}$
} 


\section{Cuadro 2. Datos de participación en elecciones presidenciales}

\begin{tabular}{|c|c|c|}
\hline Año/Elecciones & Presidenciales & Legislativas \\
\hline $1948 / 1947$ & $61,48 \%$ & $56,69 \%$ \\
\hline 1950 & & $64,11 \%$ \\
\hline 1952 & $64,91 \%$ & $69,48 \%$ \\
\hline 1954 & & $81,62 \%$ \\
\hline 1956 & $73,41 \%$ & $71,60 \%$ \\
\hline 1958 & & $48,68 \%$ \\
\hline 1960/1962 & $76,01 \%$ & $69,29 \%$ \\
\hline 1968 & $77,49 \%$ & \\
\hline 1978 & $72,83 \%$ & \\
\hline 1979 & $80,49 \%$ & $80,37 \%$ \\
\hline 1984 & $78,13 \%$ & $71,15 \%$ \\
\hline 1986 & & $74,02 \%$ \\
\hline 1988 & $77,70 \%$ & $77,65 \%$ \\
\hline 1990 & & $69,42 \%$ \\
\hline 1992/1994 & $73,10 \%$ & $65,49 \%$ \\
\hline 1996 & $71,71 \%$ & $67,87 \%$ \\
\hline 1998 & $70,13 \%$ & $47,25 \%$ \\
\hline 2002 & $62,89 \%$ & $63,51 \%$ \\
\hline 2006 & $76,01 \%$ & $63,50 \%$ \\
\hline 2009 & $75,28 \%$ & $75,72 \%$ \\
\hline 2013 & $81,06 \%$ & $81,08 \%$ \\
\hline
\end{tabular}

Fuente: International Institute for Democracy and Electoral Assistance (International IDEA)

En Ecuador la evolución histórica de la participación electoral corre paralela a la venezolana. Si bien el suelo de la participación electoral se tocó entre 1998 y 2002 (cuando la participación en las presidenciales bajó a poco más del 60\% y en las legislativas al $47,25 \%$ ), en los últimos años las tendencias de participación electoral se han recuperado con creces hasta lograr auténticos máximos históricos nunca antes alcanzados (hasta el $81 \%$ ).

Específicamente, en el caso de Ecuador, el movimiento indígena se convirtió rápidamente en un actor aglutinante de los sectores populares en un momento en que las organizaciones sindicales estaban desestructuradas y dejaban vacíos de dirección dentro de los sectores de oposición al establishment. El empuje indígena, que se articulaba desde hacía varias décadas en el ámbito local y de base, se convirtió a pesar de su heterogeneidad en un actor aglutinante 
que abogaba por construir un nuevo modelo de gestión democrática y participativa para la reconfiguración de la relación entre Estado y Sociedad. Además, el énfasis en el espacio de lo local ha facilitado la argumentación étnica y cultural (Guerrero, 1998).

La Constitución ecuatoriana de 1998 podríamos decir que evidencia el agotamiento del modelo de representación liberal anterior, ya que incorpora un nuevo lenguaje al discurso político ecuatoriano, como evidencia la inclusión de conceptos como diferencia, autonomía, plurinacionalidad, culturas, historias, racionalidad, biodiversidad, entre otros. Basta con leer el Capítulo 4 (De los derechos económicos, sociales y culturales) y especialmente el Capítulo 5 (De los derechos colectivos), en el que se incluyen derechos de los pueblos indígenas y negros o afroecuatorianos, así como derechos del medio ambiente. No obstante, el manejo de tales términos no derivó en un reconocimiento efectivo, igual que sucedió con los derechos políticos, o los sociales y culturales, esto es, se trataba en todo caso de un reformismo superficial que no logró encauzar por las vías oficiales la arraigada desafección que se acumulaba en los márgenes del sistema.

En síntesis, las formas tradicionales de participación, de corte liberal institucionalista y centradas en el sufragio, se fueron desvirtuando y perdiendo su legitimidad por culpa, en buena medida, de la praxis sostenida de los partidos políticos, y en base a tres dimensiones: en primer lugar, la pérdida de soberanía nacional-popular, debido a la subordinación de las élites locales ante los organismos político-financieros internacionales (especialmente en los últimos años, con la marcada política neoliberal). En segundo lugar la crisis de la representación y la democracia, que observamos muy claramente con la pérdida de apoyos a los partidos tradicionales al tiempo que surgían nuevas fuerzas al margen del sistema, y con la acentuada caída de la participación electoral. Por último, y quizá la dimensión más profunda en la movilización constituyente o revolucionaria, el enorme crecimiento de la pobreza, así como las desigualdades sociales, étnicas y territoriales, que afectan por igual a ambas naciones (Ortiz Crespo \& Mayorga, 2012, p.11). 


\section{Las nuevas formas de participación política: Conceptos constitucionales, análisis normativo y empírico-cuantitativo}

Las bases en las que se enraízan y sustentan las nuevas formas de participación política en estos dos países latinoamericanos se hallan principalmente en sus jóvenes Constituciones (1999 y 2008), ambas resultados de sendos procesos constituyentes que supusieron una ruptura con la tradicional forma de abordar estos procesos, generalmente a nivel exclusivo de las élites y los partidos políticos. Al contrario, estos procesos se caracterizaron por su carácter amplio y decisivamente participativo, tanto en la forma en que se llevaron a cabo como en el contenido de las Constituciones resultantes. En el siguiente cuadro recogemos los instrumentos fundamentales de participación política directa a los que dieron luz sendos procesos constituyentes:

Cuadro 3. Instrumentos constitucionales de participación política directa (CRBV 1999 y CRE 2008).

\begin{tabular}{|c|c|}
\hline \multicolumn{2}{|c|}{ Instrumentos constitucionales de participación política directa } \\
\hline ECUADOR (CRE 2008) & VENEZUELA (CRBV 1999) \\
\hline Referéndum consultivo (Art. 104) & Referéndum consultivo (Art. 71) \\
\hline $\begin{array}{l}\text { Referéndum revocatorio en todos los cargos de elección } \\
\text { popular (Art. 105) }\end{array}$ & $\begin{array}{l}\text { Referéndum revocatorio en todos los cargos } \\
\text { de elección popular (Art. 72) }\end{array}$ \\
\hline Iniciativa de creación o abrogación normativa (Art. 103) & Referéndum ratificatorio legislativo (Art. 73) \\
\hline $\begin{array}{l}\text { Iniciativa de enmienda o reforma constitucional (Arts. 103, } \\
\qquad 442 \text { y } 443)\end{array}$ & $\begin{array}{l}\text { Referéndum abrogatorio total o parcial (Art. } \\
74)\end{array}$ \\
\hline $\begin{array}{l}\text { Iniciativa de convocatoria de Asamblea Nacional } \\
\text { Constituyente (Art. 444) }\end{array}$ & $\begin{array}{l}\text { Iniciativa de enmienda constitucional (Art. } \\
\qquad 341)\end{array}$ \\
\hline Consejos de igualdad nacional (Art. 156) & $\begin{array}{l}\text { Iniciativa de reforma constitucional (Arts. 342- } \\
\text { 346) }\end{array}$ \\
\hline $\begin{array}{c}\text { Comisiones ciudadanas del Consejo de Participación } \\
\text { ciudadana y control social (Art. 209) }\end{array}$ & $\begin{array}{l}\text { Iniciativa de convocatoria de Asamblea } \\
\text { Nacional Constituyente (Art. 347-349) }\end{array}$ \\
\hline $\begin{array}{c}\text { Audiencias públicas, veedurías, asambleas, cabildos } \\
\text { populares, consejos consultivos, observatorios, y demás } \\
\text { instancias que promueva la ciudadanía -en todos los niveles } \\
\text { de gobierno- (Art. 100) }\end{array}$ & $\begin{array}{l}\text { Consejos Comunales y Comunas } \\
\text { (desarrollado por la Ley Orgánica de los } \\
\text { Consejos Comunales -LOCC- de 2009) }\end{array}$ \\
\hline
\end{tabular}


La redefinición de la participación política, así como la decidida voluntad general por hacerla efectiva constitucionalmente, fue una constante reclamada desde mucho tiempo antes. La democracia participativa era un anhelo que se concebía en global como la solución, en buena medida, a los problemas de corrupción, falta de democracia (tanto sustantiva como cualitativamente), de derechos sociales y políticos, justicia y cohesión social que sufrían ambas naciones. De esta forma, la institucionalización de la democracia participativa se concebía como la forma de trascender la desvirtuada representación y la vieja política. En esta línea, el concepto de participación, que tuvo en cuenta el

constituyente en ambos países, fue de participación continuada, directa, protagónica en el caso venezolano, es decir, acabar con el sentido accesorio que se le había dado antaño y tomarla de forma integral, transversal.

Asimismo, era también un desafío, ya que este carácter con el que se quería cimentar la nueva institucionalidad enfrentaba poderosos enemigos, ya no externos sino esencialmente internos. Nos referimos a la sedimentada mentalidad, costumbres, prácticas y formas de entender la política que se habían configurado durante décadas en un ambiente marcado por la corrupción, el clientelismo, la desafección política, la desconfianza y la escasa cultura participativa de ambas poblaciones, hablando en términos generales.

Pese a todo lo anterior, en la siguiente tabla, cuyos datos han sido extraídos del Latinobarómetro ${ }^{11}$, podemos comprobar el revulsivo democrático que supusieron estos nuevos procesos políticos y constituyentes para ambas naciones, en términos de interés por la política y satisfacción con la democracia:

${ }^{11}$ Disponibles online en: $\underline{\text { http://www.latinobarometro.org/latOnline.jsp }}$ 
Cuadro 4. Comparativa de la percepción ciudadana acerca de factores centrales para la vida democrática.

\begin{tabular}{|c|c|c|c|}
\hline Variable/País & Venezuela & Ecuador & $\begin{array}{c}\text { América Latina } \\
\text { (media) }\end{array}$ \\
\hline Interés en la política (2013) & $\mathbf{4 9 \%}$ & $\mathbf{2 8 \%}$ & $\mathbf{2 9 \%}$ \\
\hline Diferencial 1996-2013 & $+34 \%$ & $+3 \%$ & $+4 \%$ \\
\hline Apoyo a la democracia (2015) & $\mathbf{8 4 \%}$ & $\mathbf{7 0 \%}$ & $\mathbf{5 6 \%}$ \\
\hline Diferencial 1995-2015 & $+24 \%$ & $0 \%$ & $-2 \%$ \\
\hline Satisfacción con la democracia (2015) & $\mathbf{3 0 \%}$ & $\mathbf{6 0 \%}$ & $\mathbf{3 7 \%}$ \\
\hline $\begin{array}{c}\text { Importancia del voto -capacidad del voto } \\
\text { para cambiar la realidad- (2013) }\end{array}$ & $-6 \%$ & $+27 \%$ & $-1 \%$ \\
\hline Diferencial 1996-2013 & $+29 \%$ & $\mathbf{7 1 \%}$ & $\mathbf{4 0 \%}$ \\
\hline $\begin{array}{c}\text { Sentimiento de estar representado por el } \\
\text { Congreso (2015) }\end{array}$ & $\mathbf{3 1 \%}$ & $\mathbf{2 3 \%}$ & $\mathbf{2 3 \%}$ \\
\hline Cercanía con partidos políticos (2015) & $\mathbf{5 4 \%}$ & $\mathbf{2 9} \%$ & $\mathbf{4 0 \%}$ \\
\hline
\end{tabular}

Fuente: Latinobarómetro, 1995-2015.

De los informes del Latinobarómetro podemos extraer conclusiones muy ilustrativas acerca de la evolución de la situación política en ambos países y respecto de la región. En primer lugar, es en Venezuela en donde más ha crecido el apoyo a la democracia en los últimos veinte años, y en Ecuador donde más ha aumentado la satisfacción con la misma, lo cual indica la gravedad de la situación previa y el éxito relativo de los proyectos nacional-populares en el sentido de reavivar el apoyo al sistema democrático. En cuanto al interés por la política, en Venezuela se percibe más esta realidad, indicativa del grado de politización popular y, a su vez, como una de las bases necesarias para la salud de todo sistema democrático, el interés ciudadano por las cuestiones públicas. Ecuador, que se sitúa en la media del interés por la política, es en cambio el segundo de Latinoamérica en el que hay mayor grado de satisfacción con la democracia, habiendo bajado en Venezuela este indicador un brusco $12 \%$ respecto al informe de 2013. 
Otro aspecto importante de la concepción hegemónica de la democracia es la desconcentración del poder, tanto horizontal como verticalmente. Los procesos de descentralización política fueron fundamentales en ambos países, y tienen un gran interés en nuestro estudio al afectar directamente a la cuestión de la participación política, en tanto que se trata de acercar el poder y su ejercicio a la ciudadanía. En Ecuador, el discurso político predominante desde su último proceso constituyente asume la rearticulación territorial y el reconocimiento plurinacional del Estado. En el caso de Venezuela, el programa político oficialista se define federalista y aboga por una novedosa concepción comunal de la nación.

A la hora de desarrollar las nuevas formas de participación política hay que empezar por su conceptualización y definición normativa, esto es, partimos de los textos constitucionales. Sin embargo, la forma en que éstos se elaboraron es muy ilustrativa de cómo se pretende que sea la nueva institucionalidad. Como ya se mencionó antes, ambos procesos fueron más participativos que ningún otro en la historia de ambos países. En el caso de Ecuador, en la sede de Montecristi (nombre que se le da popularmente a la Constitución de 2008) se reunieron más de mil delegaciones que representaban a una gran amalgama de asociaciones, colectivos y organizaciones sociales. Durante este proceso "se implementaron una variedad de mecanismos de deliberación e información como foros, talleres, mesas itinerantes, recepción de propuestas, mecanismos virtuales, cuyo impacto alcanzó a más de cien mil personas" (Muñoz, 2008; Goldfrank, 2011, p.101; Navas, 2012b, p.98).

El desarrollo normativo-constitucional de las formas de participación política es bastante más concreto y detallado en la CRE que en la CRBV. En la primera subyace en su conjunto la idea de que la voluntad de participación popular en la vida política ha de estar consagrada y reconocida a través de derechos de participación, para los cuales se reserva un capítulo específico en el que se incorporan los derechos tradicionales o propios de la representación liberal. Además, una serie de derechos de participación activos y continuos de la 
comunidad política en las instituciones (Título II, Capítulo Quinto, arts. 61 y ss. CRE; Título IV, Capítulo Primero, Art. 95 y ss. CRE) ${ }^{12}$.

Nos centramos en la descripción de los segundos, debido a su carácter innovador respecto de los primeros. Estos, a su vez, los podemos subdividir en dos grupos; el primero, referente a la democracia participativa, se constituye de los derechos a participar en los asuntos de interés público (Art. 61.2 CRE), el mismo que guarda conexión con una serie de mecanismos y espacios institucionales que se describen más adelante, así como el derecho a fiscalizar los actos del poder público (Art. 61.5 CRE).

El segundo grupo trata los derechos relativos a las formas de ejercicio de democracia directa, que albergan el derecho a presentar proyectos de iniciativa popular normativa. Asimismo, cabe destacar un derecho que ha sido tratado de forma prolífica en la teoría política clásica, pero cuya efectividad puede resultar problemática. Se trata del derecho a la resistencia "frente a acciones u omisiones del poder público o de las personas naturales o jurídicas no estatales que vulneren o puedan vulnerar sus derechos constitucionales", y para "demandar el reconocimiento de nuevos derechos" (Título IV, Capítulo Primero, Sección Segunda, Art. $98 \mathrm{CRE}$ ).

Algunos importantes mecanismos concretos de participación son las audiencias públicas, veedurías, asambleas, cabildos populares, consejos consultivos, observatorios y las demás instancias que promueva la ciudadanía (Título IV, Capítulo Primero, Sección Tercera, Art. 100). Se instituye también el mecanismo de la silla vacía que permite a cualquier ciudadano intervenir en su propio nombre o representando a una organización, en las sesiones de los gobiernos autónomos descentralizados. De forma destacada se establecen formas de democracia directa para las comunidades, pueblos y naciones indígenas, como la iniciativa popular legislativa o normativa, la consulta popular y el instituto del

12 Constitución de la República del Ecuador, disponible en el siguiente enlace: http://www.asambleanacional.gob.ec/sites/default/files/documents/old/constitucion de bolsillo.pdf 
revocatorio de los mandatos de elección popular (Arts. 103, 104 y 105 CRE, respectivamente).

En el caso de Venezuela se establecen cuatro tipos de referéndum en los artículos 71 a 74 de la $\mathrm{CRBV}^{13}$ (aprobatorio, abrogatorio, revocatorio y consultivo) que pueden ser elevados hasta las principales instancias del Estado. Ello se puso en práctica en el referéndum revocatorio al Presidente Hugo Chávez en 2004, e igualmente es un procedimiento que actualmente está en la punta de lanza del discurso de la oposición venezolana para revocar al Presidente Maduro (una vez que ha cumplido la mitad de su mandato). Consideramos muy positivo dado el efecto disuasorio que genera respecto a otras vías de carácter inconstitucional.

Otro cambio fundamental que emergió de los procesos constituyentes fue el nuevo diseño de los poderes del Estado, que pasan de los tres clásicos (legislativo, ejecutivo y judicial) a ser cinco en Venezuela. A estos tres se le añadieron otros dos poderes, el electoral y el ciudadano, trayendo a colación el legado teórico-político de Simón Bolívar, concretamente tomando la Carta de Angostura $^{14}$ de 1819 como documento en el que sugirió tal diseño institucional ampliado $^{15}$. En el caso de Ecuador, la transformación de las funciones elementales del Estado fue similar, aunque se pueden establecer algunas distinciones. Aunque en ambos países el poder electoral sirve para garantizar la limpieza en el ejercicio de la participación política, existen diferencias entre el Poder Ciudadano en Venezuela (cuyo cometido es velar por la ética en toda la acción pública del país) y el de Transparencia y Control Social en el Ecuador. Las funciones esenciales de este último son promover el ejercicio de los nuevos derechos y las variadas formas de participación, el control de la gestión pública y el combate a la corrupción, y la designación de algunas de las más altas autoridades del Estado, bajo

\footnotetext{
${ }^{13}$ Disponible en: http://www.cne.gob.ve/web/normativa electoral/constitucion/indice.php

${ }^{14}$ Concretamente se refería a instaurar un cuarto poder aparte de los tres clásicos; el Poder Moral.

${ }^{15}$ Merece la pena recordar que algunos de los mecanismos de participación que se implementaron ya fueron abordados en la Comisión para la Reforma del Estado (COPRE) que impulsó en 1984 el Presidente Lusinchi, cuyas conclusiones no se llevaron a la práctica.
} 
procedimientos participativos en base a los méritos de los candidatos (Arts. 204208 CRE).

Como indica García-Guadilla (2006), el "objetivo que se buscaba con esta nueva arquitectura institucional era que la sociedad civil se transformara de actor social en actor socio-político y en interlocutora legítima y directa del Estado sin pasar por la mediación de los partidos políticos" (p.39). Con esta misma finalidad se diseñaron los Consejos Comunales (CC. CC.) en Venezuela, que por su carácter vanguardista en diseño, como en la dotación de recursos y el enérgico empeño con que se han puesto en práctica, considero que merecen ser estudiados con un poco más de detenimiento. Los CC. CC. responden a una forma muy cercana al ideal de la teoría democrática, de participación y poder popular en estado puro. En otras palabras, la organización política, deliberación, gestión, discusión y toma de decisiones recaen directamente en la comunidad local. Con el Estado Comunal, no solo se buscaba realizar el ideal de autogobierno, sino también la educación política, la formación práctica en labores de auto-organización, debate, gestión y toma de decisiones en común.

Siguiendo a Isidoro Losada (2015) y según indica la Ley Orgánica de los Consejos Comunales, la construcción del Estado comunal representa una nueva concepción del Estado y de la sociedad en la cual el poder es ejercido directamente por el pueblo a través de los autogobiernos comunales, "un modelo económico de propiedad social y de desarrollo endógeno y sustentable, que permita alcanzar la suprema felicidad social de los venezolanos y venezolanas en la sociedad socialista" (Losada, 2015, p.108). En esta finalidad ideológica preestablecida es donde muchos críticos han puesto sus miradas, ya que es a priori inconstitucional. Esta tesis la desarrollaremos más adelante.

Las funciones principales de los Consejos Comunales son el impulso, la planificación, implementación, y control-evaluación de proyectos y programas comunitarios. Actualmente, hay registrados más de 45.000 CC. CC. y casi 1400 Comunas, que son el escalón organizativo inmediatamente superior a los Consejos. En este ámbito se dispone de un Ministerio propio del que dependen 
directamente y en gran medida las finanzas de los Consejos Comunales, ya que se da una relación financiera directa con el poder central.

En junio de 2012, la Ley Orgánica para la Gestión Comunitaria de Competencias, Servicios y Otras Atribuciones ${ }^{16}$ les asignó a los Consejos Comunales y a otras estructuras orgánicas del poder popular amplias atribuciones en materias de políticas sectoriales como salud, infraestructura, vivienda, cultura, microcréditos y otros ámbitos de la administración pública. Se les aumentó de manera significativa el número de funciones desempeñadas por los CC. CC. y las Comunas. Además de su peso en el devenir de la política pública venezolana, los Consejos Comunales como institutos de participación política presentan las cifras más altas en la región; no obstante, no alcanzan la tasa de participación en elecciones y referendos nacionales, aunque superan con creces las de otras formas de participación política directa (Goldfrank, 2011, p.44).

En cuanto a las críticas o problemas que se achacan al funcionamiento de los Consejos Comunales, podemos clasificarlas en tres líneas amplias:

1) Falta de transparencia y reglas claras sobre su financiamiento, lo que facilita el desarrollo de la corrupción y el clientelismo en su seno. Desde el poder central se ha justificado la distribución directa de los recursos por el combate a la corrupción y del exceso de burocracia, pero de esta forma se tiende a identificar por las comunidades locales la participación en los Consejos como mero sinónimo de obtención de recursos.

2) Otro problema es la multiplicación constante de las competencias asignadas al poder comunal. Se hallan por tanto sobrecargados en una amplísima variedad de asuntos que les competen, lo cual, unido a la modesta experiencia en gestión pública de la que disponen la mayoría de sus integrantes, limita la potencial profundidad de su impacto.

3) Identificación de los Consejos Comunales como "mecanismos al servicio de viejas lógicas clientelares y como amenaza a las instituciones de la democracia

${ }^{16}$ Disponible en: http://free-news.org/PDFs/Venezuela LO-Gestion-Comunitaria.pdf 
representativa" (Goldfrank, 2011, p.41). Asimismo y como se indicó anteriormente, la concepción de los Consejos Comunales como instrumentos para la construcción de la "sociedad socialista" que propugna el Gobierno nacional, es una contradicción teórica esencial con el diseño participativo, libre y deliberativo del poder comunal.

Respecto a las nuevas formas de participación política en Ecuador, el proyecto político dirigido por Rafael Correa y Alianza País ha incidido en el propósito de rediseñar las bases del Estado desde una perspectiva participativa y republicana. Esta tendencia se identifica claramente en la CRE mediante un conjunto de visiones sobre lo que podría ser un orden político basado en la participación continua y activa de los ciudadanos en su autogobierno. En tal contexto, el principio de participación directa configura toda la organización del Estado y es transversal a los derechos.

En conjunto, tanto los derechos tradicionales como los nuevos derechos de participación, buscan garantizar una nueva relación entre los ciudadanos y el manejo del Estado en el marco de un sistema más complejo (mixto o heterodoxo podría decirse) de democracia representativa participativa y directa. Este reconocimiento de derechos permite la transversalidad del componente participativo en todos los ámbitos de la actividad pública, destacando especialmente el ámbito local. A la vez, el ya referido sistema amplio de garantías jurisdiccionales, de política pública y normativas, refuerza la posibilidad de ejercicio de estos derechos y sus mecanismos. Como mecanismos se reconocen todas las formas de organización social necesarias para desarrollar "procesos de autodeterminación", incidir en las decisiones públicas y ejercer el control social en todos los niveles de gobierno y entidades que presten servicios públicos (Art. 96, CRE). Esta breve relación de las normas constitucionales es suficiente para comprender que "el principio participativo que la Carta prohíja es transversal y expansivo" (Montaña Pinto, 2012, p.147). 


\section{Conclusiones: valoración global y desafíos}

Como conclusiones globales hallamos que, sobre todo en sus primeros años, estos procesos revolucionarios nacional-populares supusieron una inyección de ilusión y motivación colectiva hacia lo público de amplias capas de la población. En este sentido, contribuyeron a la renovación y relegitimación de la infraestructura estatal, como al aumento de la participación política. En este período asistimos a una tendencia desarrollista de sus economías y las redes de protección social en temas esenciales como educación, salud, seguridad social, vivienda, cultura o transporte.

En cuanto a las conclusiones específicas sobre los objetivos planteados al inicio, encontramos en primer lugar que la situación económica y socio-política en ambos países viene deteriorándose en los últimos tiempos. En el caso de Venezuela, en la dimensión política, concluimos que el poder Comunal se halla fuertemente contaminado por las lógicas clientelares y dirigistas con que se ha gestionado tal proyecto político. También, tras el fallecimiento de Hugo Chávez, existe un vacío de autoridad y una caída de la confianza ciudadana que se ha traducido en una situación de ingobernabilidad. Se ha producido un profundo y acelerado deterioro político y económico del país que ya está conllevando grandes cambios en el proceso político venezolano. Esta situación se vio reflejada en las pasadas elecciones del 6 de diciembre de 2015, en que los resultados arrojan un rechazo mayoritario al chavismo como proyecto de país, en beneficio de una oposición que se halla por primera vez desde hacía 18 años con mayoría cualificada de dos tercios en la Asamblea Nacional.

En la dimensión socio-económica, el panorama en Venezuela es desolador y afecta cotidianamente al conjunto del país, en todos sus ámbitos. Aspectos como la inseguridad, la escasez, la inflación, y la extrema polarización política, desvirtúan en la práctica los mecanismos de participación ciudadana, por muy avanzados que pudieran parecernos sobre el papel. En su defecto, se transgreden en una suerte de instrumentos clientelares que, como los Consejos Comunales o los recientemente impulsados CLAP (Comités Locales de Abastecimiento y 
Producción), son concebidos por buena parte de la ciudadanía como espacios de transacción de recursos para satisfacer sus necesidades básicas a cambio de sumisión política. Todas estas penurias se reflejan, por ejemplo, en el abrupto descenso de los porcentajes de satisfacción y calidad democrática que muestran informes como el Latinobarómetro o el IDD-LAT.

En el caso de Ecuador, consideramos elogiable que el proceso constituyente de 2008 fuera un espacio político altamente participativo, pero también encontramos una erosión del fervor participativo existente en los momentos constituyentes. Ello está relacionado, entre otros aspectos, con las notables dificultades económicas que atraviesa el país (y en general la región) debido a la caída internacional de los precios de las materias primas, fuente principal de ingresos nacionales, y que se achacan a una errática política gubernamental de profundización del modelo extractivista. La crisis económica, sumada a las catastróficas consecuencias humanas y materiales que provocó el terremoto del pasado mes de abril, ha llevado según el discurso gubernamental a la necesidad de realizar fuertes recortes presupuestarios y subidas de impuestos. Esto viene generando en la población un amplio malestar y una creciente polarización que ponen en riesgo el desarrollo del proyecto participativo de país establecido en la CRE.

En ambas constituciones, cuyas influencias recíprocas son notables, las dimensiones garantistas y de participación tenían como horizonte el desarrollo autónomo de una sociedad civil fuerte que pudiera convertirse en real protagonista de una nueva forma de Estado verdaderamente democrática. Tal y como se desprende del análisis comparativo, extraemos dos conclusiones; en primer lugar, que teniendo en cuenta la situación de la que emergieron sendos procesos constituyentes (crisis de confianza en la representación política y amplias capas populares afectadas por la pobreza), así como la crisis económica actual y el bajo índice de población que se siente representado por instituciones como el Congreso, se corre actualmente el riesgo de volver a una situación similar a la anterior (constatando así el fracaso de sendas revoluciones). Por otro lado, se 
presenta la tesitura apropiada para potenciar los nuevos instrumentos de participación política, priorizando su labor en el campo socio-económico, en la configuración de formas económicas alternativas al extractivismo en favor de un desarrollo sostenible e inclusivo. Para ello, además de fraguar una separación nítida de participación ciudadana y lógicas partidistas, será necesario un amplio y constante esfuerzo de formación política de la ciudadanía, atendiendo además al papel central otorgado al estrato judicial, pues el diseño constitucional vigente es extenso, complejo y eventualmente difícil de implementar sin tales premisas.

En el caso venezolano, habrá que seguir atentamente la evolución de la economía y el desarrollo de los acontecimientos políticos, con la esperanza de una normalización de esos ámbitos que posibilite un efectivo desarrollo de la participación política, y una normalización de la vida democrática y el pluralismo en la sociedad venezolana. En el caso de Ecuador, dada la juventud del texto constitucional, también consideramos fundamental para el futuro de estas líneas de investigación el seguimiento del desarrollo de los ambiciosos instrumentos participativos, tanto lo urbano como en lo rural. Además, una especial atención a la innovadora concepción de la plurinacionalidad indígena y a la acción de la justicia constitucional, clave para la efectividad del amplio catálogo de derechos socio-políticos reconocido.

\section{Referencias Bibliográficas}

Ávila Linzán, L. F. (2012). Política, justicia y Constitución. Quito: CEDEC.

Ayala Mora, E. (2003). Centralismo y descentralización en la historia del Ecuador. Del pasado a la situación actual. Procesos, Revista Ecuatoriana de Historia, 19, 203-221.

Banco Interamericano De Desarrollo (2015). Construyendo gobiernos efectivos. Resúmenes ejecutivos de los informes nacionales.

Banco Mundial (2015). Comunicado de prensa, 6 de octubre, Lima (Perú).

Bonometti, P. y Ruiz Seisdedos, S. (2010). La democracia en América Latina y la constante amenaza de la desigualdad. Andamios, 7(13), 11-36.

Central Intelligency Agency (2016). The World Factbook.

Constituciones Políticas de la República del Ecuador (2008, 1998 y 1978)

Constitución Política de la República Bolivariana de Venezuela (1999).

Constitución de la República de Venezuela (1961). 
Cordero Heredia, D. (2009). Nuevas instituciones del Derecho constitucional ecuatoriano. Quito: INREDH.

Freidenberg, F. (2012). Ecuador 2011: Revolución Ciudadana, estabilidad presidencial y personalismo político. Revista de Ciencia Política, 32(1), 129-150.

García-Guadilla, M. P. (2006). Organizaciones sociales y conflictos sociopolíticos en una sociedad polarizada: las dos caras de la democracia participativa en Venezuela. Participación ciudadana, 42, 37-60.

Gargarella, R. (2012). La justicia frente al gobierno. Sobre el carácter contramayoritario del poder judicial. Quito: CEDEC.

Goldfrank, B. (2011). Los Consejos Comunales: ¿Avance o retroceso para la democracia venezolana? Íconos. Revista de Ciencias Sociales, 39, 41-55.

Gott, R. (2005). Hugo Chávez and the Bolivarian Revolution. London New York: Verso Books.

Guerrero, A. (1998). Ciudadanía, frontera étnica y compulsión binaria. Íconos. Revista de Ciencias Sociales, 4, 112-124.

Instituto Nacional de Estadística (2016). Serie histórica de pobreza por ingreso, 1997-2013. MPPP, Gobierno Bolivariano de Venezuela.

Isidoro Losada, A. N. (2015). Estrategias territoriales de control político en Venezuela. Freie Universität Berlin, Alemania Iberoamericana, XV, 59, 157-170. Ley Orgánica de Participación Ciudadana. Registro oficial. 12 de abril de 2010. Quito, Ecuador. Ley Orgánica de los Consejos Comunales. Gaceta Oficial de la República Bolivariana de Venezuela. 26 de noviembre de 2009, Caracas, Venezuela.

López Maya, M. (2015). Participación y poder popular en Venezuela: antes y ahora. Revista de Sociedad, Cultura y Política en América Latina, 3(3), 32-45. Montaña Pinto, J. (2012). Teoría utópica de las fuentes del derecho ecuatoriano. Perspectiva comparada. Quito: CEDEC.

Muñoz, J. P. (2008). Movimientos sociales y procesos constituyentes. El caso de Ecuador. Institut de recherche et débat sur la gouvernance (IRG).

Navas Alvear, M. (2014). Proceso constituyente, participación y un nuevo diseño democrático en la constitución ecuatoriana actual. Universidad Andina Simón Bolívar, 6(2), 94-116.

Núñez Sánchez, J. (2014). Ecuador. Revolución Ciudadana y Buen Vivir. Barcelona: Yulca.

Ortiz Crespo, S. \& Mayorga, F. (2012). Movimientos sociales, Estado y democracia en Bolivia y Ecuador en el tránsito del neoliberalismo al postneoliberalismo. íconos. Revista de Ciencias Sociales. 44, 11-17.

Ramírez Gallegos, F. (2011). Primera encuesta nacional sobre jóvenes y participación política en Ecuador. Flacso Ecuador. 
Rangel Cruz, P. A. (2012). Ideología, acción política y sociedad civil. El correlato del poder centralizado en Venezuela. Compendium: revista de investigación científica, 29: 51-66.

Senplades (2011). Tendencias de la participación ciudadana en el Ecuador. Uriarte, E. (2008). Introducción a la Ciencia Política: La política en las sociedades democráticas. Madrid: Tecnos.

Welsh, F. \& Carrasquero, J. V. (1996). ¿Desconsolidación de la democracia en Venezuela? Rendimiento y legitimidad normativa. Cuestiones Políticas, 16, 45-69.

\section{Para citar este artículo:}

Criado, J. (2016). Análisis socio-histórico comparado sobre las formas de participación política en Venezuela y Ecuador. Collectivus, Revista de Ciencias Sociales, $\quad 3(2), \quad 58-84 \quad$ doi: $\quad$ http://dx.doi.org/10.15648/Coll.2.2016.4 